\title{
Festschrift in honor of Oliviero Stock_—— Preface
}

\section{Marco Gori $^{1}$ (D) Ramon Lopez de Mantaras ${ }^{2}$. Carlo Strapparava ${ }^{3}$}

Published online: 6 February 2020

(C) Springer Nature Switzerland AG 2020

Oliviero Stock was born on 5-5-50. When he turned 55, he involved a number of friends in the game of sending him a message with something interesting concerning number 5 . He wanted to stick to $5^{2}$ people, but the number quickly broke this threshold. His mailbox, and that of people involved in the game, was soon flooded with comments on 5 that came from mathematicians, AI researchers, philosophers, computational linguists, cognitive scientists, and historians.

Amongst others, it was pointed out that the secrets of his 5-5-50 is nicely related to the golden ratio $\phi$ in Fibonacci sequences $F_{n}=F_{n-1}+F_{n-2} ; F_{0}=0, F_{1}=1$. The general solution is based on $\phi=(1+\sqrt{5}) / 2$. Surprisingly enough it can be rewritten as $\phi=.5 * .5^{5}+.5$ Interestingly, we have $F_{5}=5$, and for large $n$, we have $F_{n} \rightarrow \phi^{n} / \sqrt{5}$. No doubt, 5 is a fundamental ingredient in $\phi$, the golden ratio number, the Divine proportion. The historic book on arithmetics, Liber Abaci, by Leonardo Pisano, posthumously known as Fibonacci, introduced the number $F_{n}$ to explain the rabbit mating problem, as Fibonacci's numbers are somewhat related to reproduction.

When his parents chose a first name for their youngest son, the beauty of Fibonacci's recursion prevailed: Looking at 5-5-50 they must have focussed on $F_{5}=5$, since their family name "Stock" is composed of 5 characters, and when thinking of the next generation, they were forced to choose a name with 8 characters, since the next Fibonacci number is $F_{6}=8$. Of course, "Oliviero" is 8 characters! No wonder-even his name is part of the mystery of the Number Five.

Carlo Strapparava

strappa@fbk.eu

Marco Gori

marco@dii.unisi.it

Ramon Lopez de Mantaras

mantaras@iiia.csic.es

1 University of Siena, Siena, Italy

2 Artificial Intelligence Research Institute, Bellaterra, Spain

3 FBK-irst, Trento, Italy 


\section{The Pleasure of Research in AI}

On of April 2018, the Fondazione Bruno Kessler (FBK) in Trento, Italy organized a workshop on "The Pleasure of Research in AI" to celebrate 30 years of research in artificial intelligence at FBK-irst, and dedicated to Oliviero Stock, former director of IRST. In his talk, Oliviero clearly couldn't refrain from spreading the magic of five. The title Ay Eye Oy $O y$ ! was on two lines, with two words each whose overall length summed up to 5.

Seventeen researchers who have worked with Oliviero Stock over the years, presented lectures at the workshop, including five invited plenary speakers from abroad. Ron Brachman described Why description logics were so much fun. Martin Golumbic gave a talk on Mathematics and artificial intelligence, highlighting the topics on which Oliviero has contributed. Barbara Grosz spoke on Smart enough to talk with us? Foundations and challenges for dialogue capable AI systems. Candy Sidner lectured on Challenges in teaching robots to perform tasks. Wolfgang Wahlster told the audience about The joy of multimodal discourse. Italian colleagues who gave talks were Luigia Carlucci Aiello, Cristiano Castelfranchi, Rino Falcone, Giacomo Ferrari, Marco Gori, Bruno Lepri, Bernardo Magnini, Fabio Pianesi, Fancesco Ricci, Niculae Sebe, Carlo Strapparava, and Massimo Zancanaro

\section{The Festschrift}

As this special issue of the Annals of Mathematics and Artificial Intelligence appears on the 5th of May 2020, $(\mid$ Oliviero $|$,$| Stock \mid)=\left(F_{6}, F_{5}\right)$ will turn $70=F_{5}^{2} F_{6}-F_{5}^{3}-F_{5}$. Since the exponents also sum up to 5 , it looks like his $5 \times 5+5 \times 5+5 \times 5-5$ years, confirms his being fully deserving this Festschrift.

Oliviero Stock is a pioneer in many challenging domains in artificial intelligence such as Multimodality and NLP, Computational Humour, Computational Creativity, Persuasive NLP, and AI Ethics. His unique view of artificial intelligence is that of a computational technology that helps humans, both directly and indirectly, to improve the quality of their lives. In this special issue of AMAI, we welcomed papers from all areas of artificial intelligence, and especially from those topics in which Oliviero has been active.

Regarding computational humour, the paper "All the world's a stage: incongruity humor revisited" by Anton Nijholt offers an interesting and comprehensive survey of the philosophy of humour, focusing mainly on work prior to the 20th century, but also including landmarks in that century and more recent developments.

On creativity, and how AI can deal with it, this special issue includes several interesting papers. "Changing channels: divergent approaches to the creative streaming of texts" by Tony Veale presents an overview of the author's work on metaphor generation. The main point is that the web offers a variety of linguistic channels, each with its own characteristic properties. These different channels can be leveraged to produce different forms of metaphorical texts. The paper showcases a very broad set of scenarios in which a sensible combination of lexical resources, repositories of world and common sense knowledge, and statistical methods can be successfully employed to generate compelling and amusing texts.

"Towards a model of creative understanding" by Zoe Falomir and Enric Plaza addresses computational models of novelty and creativity from the viewpoint of conceptual blending theory. Specifically, the paper focusses on novelty (a new blend, a new concept) in a communication setting, where the receiver agent recreates the blend using its own mental spaces. 
In "Blending under deconstruction: the roles of logic, ontology, and cognition in computational concept invention", Roberto Confalonieri and Oliver Kutz present a coherent computational narrative on how to reconstruct formally conceptual blending aspects. In particular, they focus on the construction of a generic space and on the evaluation of generated blends by means of refinement operators.

Oliviero coordinated many research projects, including the ice-breaking European project HAHAcronym on reanalysis and generation of humorous acronyms. In this special issue, "Acronyms: identification, expansion and disambiguation" by Kayla Jacobs, Alon Itai and Shuly Wintner presents a machine-learning based approach to automatically extract an acronym dictionary from unannotated texts.

Specifically on natural language processing, the paper "Dual embeddings and metrics for word and relational similarity" by Dandan Li and Douglas Summers-Stay addresses the problem of word and relational similarity. The technique consists of combining two different word embeddings, obtained from state-of-the-art methodologies that are known to capture different properties of a word (functional and topical), and different metrics to measure word and relational similarity.

Exploring communication channels beyond pure natural language, "A computer agent that develops visual compositions based on the ER-model" by Rafael Pérez y Pérez and Iván Guerrero Román describes a computer agent for the automatic generation of visual compositions based on the Engagement-Reflection Model of creative writing.

"Spatial reasoning about qualitative shape compositions: composing qualitative lengths and angles" by Zoe Falomir, Albert Pich and Vicent Costa deals with the topic of qualitative spatial and temporal representations and reasoning. In particular, the paper proposes a qualitative model for angles and length, based on the temporal model proposed by Allen, and prove their composition relations and correctness.

More on the side of logic, "Non-terminating processes in the situation calculus" by Giuseppe De Giacomo and Eugenia Ternovska describes a situation calculus approach to expressing and proving properties of non-terminating programs expressed in GOLOG, a high level logic programming language for modeling and implementing dynamical systems.

Finally, planning is covered by the paper "Discovering state constraints for planning with conditional effects in DISCOPLAN" by Alfonso Gerevini and Lenhart Schubert. The paper presents an overview, and detailed algorithms, for discovering state constraints in planning domains via DISCOPLAN, a domain-independent approach able to infer and exploit invariants in domain models.

As editors of this special issue, we think that these papers provide a good picture of Artificial Intelligence, especially on those topics to which Oliviero devoted his brilliant research activity. Congratulations Oliviero!

\author{
Guest editors \\ Marco Gori \\ Ramon Lopez de Mantaras \\ Carlo Strapparava
}

Publisher's note Springer Nature remains neutral with regard to jurisdictional claims in published maps and institutional affiliations. 